\title{
NEW AND OLD PROBLEMS FOR ENTIRE FUNCTIONS
}

\author{
LOUIS DE BRANGES
}

An entire function is a function which is defined and differentiable in the complex plane. Although there is an extensive classical theory of such functions, they are now best known for their occurrence in the theory of integral transformations. The first problem is therefore to obtain integral representations of entire functions.

The representation theory of entire functions as $L^{2}$ Fourier transforms is due to Paley and Wiener [18]. Consider the Fourier representation of a function

$$
F(x)=\int f(t) e^{i x t} d t
$$

in $L^{2}$ when its Fourier transform $f(x)$ vanishes outside of some finite interval $[-a, a]$. In this case, if $F(x)$ is suitably redefined in a set of measure zero, it is the restriction to the real axis of an entire function $F(z)$ which satisfies the inequality

$$
|F(x+i y)|^{2} \leqq \int|F(t)|^{2} d t\left(e^{2 a y}-e^{-2 a y}\right) /(4 \pi y)
$$

in the complex plane. Paley and Wiener show that an entire function which satisfies this inequality and is square integrable on the real axis is the Fourier transform of an $L^{2}$ function which vanishes outside of $[-a, a]$. Actually they show more, but this is a technical point for which I refer to Boas's book on entire functions [2].

The significance of the theorem is that it reduces the study of the $L^{2}$ Fourier transformation to the study of entire functions which are square integrable on the real axis. Corresponding to any given number $a>0$, there is a Hilbert space of entire functions, characterized by an inequality in the complex plane. These Paley-Wiener spaces are contained isometrically in $L^{2}$. They are totally ordered by inclusion. Their union is dense in $L^{2}$ and their intersection contains no nonzero element. A knowledge of these spaces is sufficient to determine the Fourier transformation. The problem now is to obtain a similar characterization of functions which are representable for other integral transformations.

An address delivered before the Madison meeting of the Society on November 30, 1963 by invitation of the Committee to Select Hour Speakers for Western Sectional Meetings; received by the editors November 30, 1963. 
An admissible transformation must obviously bear a close relationship to the Fourier transformation. Now the Fourier transformation may be looked upon as the eigenfunction expansion for the selfadjoint operator $-i d / d x$ in $L^{2}$, and it is closely related to the eigenfunction expansion for $-d^{2} / d x^{2}$. It is natural to look at the eigenfunction expansions for more general selfadjoint, second-order differential operators in $L^{2}$. A general discussion of such expansions is given by $\mathrm{E}$. C. Titchmarsh [21], and it is only necessary to rephrase his discussion in the language of entire functions, so as to generalize the Fourier situation.

My approach to the problem is based upon the work of M. H. Stone [20]. Stone's analysis of second-order differential operators depends on the theory of symmetric transformations in Hilbert space. A linear transformation $H$ in a Hilbert space is said to be symmetric if the relation $\langle H f, g\rangle=\langle f, H g\rangle$ holds whenever it makes sense - that is, whenever $f$ and $g$ are in the domain of $H$. A symmetric transformation is like a selfadjoint transformation, but differs from it in that the inverse of $H-w$, which exists when $w$ is not real, need not be densely defined. This fact is responsible for the connection between symmetric transformations and complex function theory.

I make this connection by considering Hilbert spaces, whose elements are entire functions, and which have these properties:

(H1) Whenever $F(z)$ is in the space and has a nonreal zero $w$, the function $F(z)(z-v \bar{w}) /(z-w)$ is in the space and has the same norm as $F(z)$.

(H2) For every nonreal number w, the linear functional defined on the space by $F(z) \rightarrow F(w)$ is continuous.

(H3) The function $F^{*}(z)=\bar{F}(\bar{z})$ is in the space whenever $F(z)$ is, and it always has the same norm as $F(z)$.

Now consider a transformation, multiplication by $z$ in the given space, which takes $F(z)$ into $z F(z)$ whenever $F(z)$ and $z F(z)$ are in the space. The axiom (H1) says that multiplication by $z$ is symmetric and has deficiency index $(1,1)$. The axiom (H2) says that multiplication by $z$ is a closed transformation. The axiom (H3) says that multiplication by $z$ is real with respect to a conjugation which coincides with complex conjugation of function values on the real axis. It turns out that there is a close relationship between such transformations and the theory of entire functions in Boas's book [2].

Boas studies entire functions in relation to a line, which is conveniently chosen as the real axis. His principal tool is a representation theorem for analytic functions in a half-plane. In the case of entire functions, this representation amounts to a factorization into a 
Blaschke product and an entire function which has no zeros in the upper half-plane $(y>0)$. A fundamental concept in his work is that of an entire function which has no zeros in the upper half-plane. He secures a zero-free half-plane by requiring that

$$
|E(x-i y)|<|E(x+i y)|
$$

for $y>0$. An entire function of exponential type necessarily satisfies this inequality if it has no zeros for $y>0$ and if it satisfies some general condition of growth. For example, if $E(z)$ and $E^{*}(z)$ are linearly independent, it is sufficient to have

$$
\lim \sup y^{-1} \log |E(-i y)| \leqq \lim \sup y^{-1} \log |E(i y)|
$$

as $y \rightarrow+\infty$.

If $E(z)$ is an entire function which satisfies this inequality, I will write $E(z)=A(z)-i B(z)$, where $A(z)$ and $B(z)$ are entire functions which are real on the real axis, and

$$
K(w, z)=[B(z) \bar{A}(w)-A(z) \bar{B}(w)] /[\pi(z-\bar{w})] .
$$

Let $\mathfrak{H C}(E)$ be the set of entire functions $F(z)$ such that

$$
\|F\|^{2}=\int|F(t) / E(t)|^{2} d t<\infty
$$

with integration on the real axis, and such that

$$
|F(z)|^{2} \leqq\|F\|^{2} K(z, z)
$$

for all complex $z$. Then $\mathfrak{H C}(E)$ is a Hilbert space of entire functions which satisfies (H1), (H2), and (H3). For each complex number w, $K(w, z)$ belongs to $\mathcal{H C}(E)$ as a function of $z$ and

$$
F(w)=\langle F(t), K(w, t)\rangle
$$

for every $F(z)$ in $\mathcal{H}(E)$. A Hilbert space, whose elements are entire functions, which satisfies (H1), (H2), and (H3), and which contains a nonzero element, is equal (isometrically) to $\operatorname{HC}(E)$ for some such entire function $E(z)$.

For example, let $E(a, z)=e^{-i a z}$, where $a>0$. Then, $A(a, z)=\cos (a z)$, $B(a, z)=\sin (a z)$, and

$$
K(a, w, z)=\frac{\sin (a z-a \bar{w})}{\pi(z-\bar{w})} .
$$

(The index $a$ is written as a new variable to avoid awkward subscripts.) The space $\mathcal{F C}(E(a))$ is the space of entire functions $F(z)$ such that 


$$
\|F\|^{2}=\int|F(t)|^{2} d t<\infty,
$$

since $E(a, z)$ has absolute value 1 on the real axis, and such that

$$
|F(x+i y)|^{2} \leqq\|F\|^{2}\left(e^{2 a y}-e^{-2 a y}\right) /(4 \pi y)
$$

for all complex $z=x+i y$. In other words, $\Re(E(a))$ is the PaleyWiener space of $L^{2}$ functions whose Fourier transforms vanish outside of $[-a, a]$. An entire function $F(z)$ belongs to $\mathfrak{H C}(E(a))$ if, and only if, it can be represented as

$$
F(z)=\int e^{i z t} f(t) d t,
$$

where $f(x)$ belongs to $L^{2}$ and vanishes outside of $[-a, a]$.

If an entire function $F(z)$ belongs to the space $\mathfrak{F C}(E(a))$, the Parseval relation for Fourier integrals can be applied to give

$$
\int|F(t)|^{2} d t=2 \pi \int_{-a}^{a}|f(t)|^{2} d t .
$$

The Parseval relation can also be applied to the Fourier series expansion of $f(x)$ in $L^{2}(-a, a)$, and this gives

$$
\frac{\pi}{a} \sum\left|F\left(\frac{n \pi}{a}\right)\right|^{2}=2 \pi \int_{-a}^{a}|f(t)|^{2} d t .
$$

It follows from these two formulas that

$$
\int|F(t)|^{2} d t=\frac{\pi}{a} \sum\left|F\left(\frac{n \pi}{a}\right)\right|^{2},
$$

where the summation is taken over the integers. The formula is used by Boas [2], who deduces it, as I have just done, by combining the $L^{2}$ theory of Fourier series with the $L^{2}$ theory of Fourier integrals.

My work on Hilbert spaces of entire functions originates in the discovery [8] that this formula has a simple direct proof which is independent of Fourier analysis. The formula is a special case of a general formula whose statement requires a space $\mathfrak{H C}(E)$. (That is how the space was discovered.) To state the formula, I will suppose that $\phi(x)$ is a continuous determination of the phase of $\bar{E}(x)$. Then $\phi(x)$ is an increasing function of real $x$ which has a positive derivative everywhere. The formula is

$$
\int|F(t) / E(t)|^{2} d t=\sum \frac{\pi}{\phi^{\prime}(t)}|F(t) / E(t)|^{2},
$$


where the summation on the right is over all real $t$ such that $\phi(t)$ $\equiv \alpha$ (modulo $\pi$ ). With the possible exception of at most one value of $\alpha$ modulo $\pi$, this formula is valid for every $F(z)$ in $\mathcal{K C}(E)$.

We have seen that the formula is closely related to the theory of the $L^{2}$ Fourier transformation in the case that $E(z)=e^{-i a z}$. In this case it is an expression of the relationship between Fourier series and Fourier integrals. That a similar formula is valid for a general space $\mathfrak{F C}(E)$ suggests that there should be some generalization of the Fourier transformation associated with such a space. Such a transformation always does exist, and is obtained by considering spaces $\mathcal{H C}(E(a))$ which are contained isometrically in the given $\mathfrak{K}(E)$.

To avoid minor technical difficulties, I will suppose that the functions $E(z)$ and $E(a, z)$ have no real zeros. Under this hypothesis, the spaces $\mathfrak{H}(E(a))$ contained isometrically in $\mathfrak{H}(E)$ are totally ordered by inclusion. That is, if $\mathfrak{F}(E(a))$ and $\mathfrak{H C}(E(b))$ are contained isometrically in $\mathfrak{F C}(E)$, and if $E(a, z)$ and $E(b, z)$ have no real zeros, then either $\mathfrak{F C}(E(a))$ must contain $\mathfrak{H}(E(b))$ or $\mathfrak{H}(E(b))$ must contain $\mathfrak{H}(E(a))$. Furthermore, there always exist spaces $\mathfrak{F}(E(a))$ contained properly in any given $\mathfrak{F C}(E)$ if $\mathfrak{H C}(E)$ is not one-dimensional. The family of such spaces is associated with a first-order differential operator in much the same way as the Paley-Wiener spaces are associated with $-i d / d x$. The eigenfunction expansion for the differential operator is then the required integral representation.

The precise formulation of these expansions is necessarily complicated because of its generality. It is found in my Transactions papers [10-13] on Hilbert spaces of entire functions. As a special case the theory contains a new and more precise eigenfunction expansion for second-order differential operators.

A second fundamental problem in the theory of entire functions is the problem of polynomial approximation and, more generally, the problem of approximation by entire functions of exponential type. Among such problems the Bernstein problem on weighted polynomial approximation is one of the most interesting. This problem should be thought of in relation to the Weierstrass approximation theorem and M. H. Stone's generalization of it. It is now well known that the theorem is closely related to the Krein-Milman theorem on extreme points of compact, convex sets. What is not so well known is that the same relationship persists between the KreIn-Milman theorem and the problem of weighted polynomial approximation.

My formulation of the problem follows that of H. Pollard [19]. Let $w(x)$ be a continuous function of a real variable, with positive values, such that the product $P(x) w(x)$ remains bounded on the real axis for every choice of polynomial $P(x)$. The problem is to determine what 
functions $f(x)$ can be approximated uniformly on the real axis by such weighted polynomials $P(x) w(x)$. Such functions $f(x)$ are necessarily continuous and have limit zero at infinity, and I will confine myself to functions with these properties. By the Hahn-Banach theorem, $f(x)$ is the uniform limit of weighted polynomials if, and only if, $\int f(t) d \mu(t)=0$ whenever $\mu(x)$ is a function of bounded variation such that $\int P(t) w(t) d \mu(t)=0$ for every polynomial $P(x)$. It is obviously sufficient to confine oneself here to the convex set of such functions $\mu(x)$ which are real valued and have total variation at most 1 . By a theorem of Krein and Milman, the set of all such functions $\mu(x)$ is determined by its extreme points. It turns out that the extreme functions have many interesting special properties. They are always step functions with an infinite number of jumps. The jumps for any given extreme choice of $\mu(x)$ occur at the zeros of an entire function of minimal type. This means in particular that the jumps form a sequence of zero density. A consequence is that $f(x)$ can be approximated uniformly on the whole line by weighted polynomials if, and only if, it can be approximated uniformly by weighted polynomials on every real sequence with density zero [7]. Similar results have been obtained independently by P. Malliavin [17], using methods of potential theory.

A third fundamental problem in the theory of entire functions is the problem of local operators. The problem is due to H. Pollard and is the subject of my thesis [4]. If $K(x)$ is a measurable function of real $x$, define a corresponding operator $K(H)$ on absolutely convergent Fourier transforms,

$$
f(x)=\int e^{i x t} d \mu(t)
$$

by

$$
K(H) \cdot f(x)=\int e^{i x t} K(t) d \mu(t)
$$

whenever

$$
\int|K(t) d \mu(t)|<\infty .
$$

The operator $K(H)$ is said to be local if whenever two functions $f(x)$ and $g(x)$ in the domain of the operator agree in the neighborhood of some point $x_{0}$, then $K(H) \cdot f(x)$ and $K(H) \cdot g(x)$ agree in a neighborhood of $x_{0}$. For example the operator $K(H)$ is local if $K(z)$ is an entire 
function of minimal exponential type. Conversely, if the operator $K(H)$ is local and if it contains in its domain two different functions which agree in the neighborhood of a point, then $K(x)$ is the restriction to the real axis of an entire function of minimal type which satisfies

$$
\int\left(1+t^{2}\right)^{-1} \log ^{+}|K(t)| d t<\infty .
$$

A consequence is that an entire function $K(z)$ of minimal exponential type, which does not satisfy (1), determines an operator which has in its domain no nonzero function which vanishes in an interval. This fact is of interest in connection with the work of N. Levinson [16] on Fourier transforms of nonvanishing functions. The theory of local operators leads to a new and simple proof of Levinson's results. It also allows them to be extended in the following way. If $K(x)$ is a measurable function of a real variable which does not satisfy (1) and if $\log |K(x)|$ is uniformly continuous, then there exists no nonzero function in the domain of $K(H)$ which vanishes in any interval. A consequence is the theorem that an absolutely convergent Fourier transform $\int e^{i x t} d \mu(t)$, which is not identically zero, cannot vanish in any interval if there are too many gaps in the support of the measure determined by $\mu(x)$. A sufficient condition for this is that $\mu(x)$ is constant in each interval $\left(a_{n}, b_{n}\right)$ of a sequence of disjoint intervals to the right of $x=1$, whose lengths $b_{n}-a_{n}$ are bounded away from zero, and which satisfy

$$
\sum\left(b_{n}-a_{n}\right)^{2} /\left(a_{n} b_{n}\right)=\infty .
$$

If $a$ is a given positive number, the operator $K(H)$ is said to be $a$-local if whenever two functions $f(x)$ and $g(x)$ in the domain of $K(H)$ agree in a neighborhood $[h-a, h+a]$ of a point $h$, then $K(H) \cdot f(x)$ and $K(H) \cdot g(x)$ agree at $h$. If the operator $K(H)$ is $a$-local and if it contains in its domain two different functions which agree in an interval $[h-a, h+a]$, then $K(x)$ is the restriction to the real axis of an entire function $K(z)$ of exponential type at most $a$ which satisfies (1). The converse, however, is false. An entire function $K(z)$ of exponential type $a$ which satisfies (1) need not define an $a$-local operator. An unsolved problem is to determine what additional conditions are needed on $K(z)$ to yield an $a$-local operator. A sufficient condition is that

$$
e^{-a|y|} K(i y)=o(y)
$$

as $|y| \rightarrow \infty$. This condition is known to be necessary if 


$$
\sum\left(1+n^{2}\right)^{-1}\left|K\left(\frac{n \pi}{a}\right)\right|<\infty
$$

Some typical properties of $a$-local operators are satisfied if $K(z)$ satisfies some smoothness condition on its modulus, for instance if

$$
\frac{\partial}{\partial y} \frac{y}{\pi} \int \frac{\log \left[1+|K(t)|^{2}\right] d t}{(t-x)^{2}+y^{2}} \leqq c<\infty
$$

is bounded above for $y>0$. But the operator $K(H)$ need not be $a$-local under this hypothesis.

The $a$-local operator problem can be reformulated as an approximation problem for entire functions of exponential type and methods from the Bernstein problem can be applied. The extreme point method leads to a reformulation of the problem in terms of infinite partial fraction decompositions.

An interesting new contribution to local operators was recently made by Beurling and Malliavin [1]. Suppose that the operator $K(H)$ is $a$-local and that it has in its domain a function which vanishes in an interval of length $2 a$ and which does not vanish identically. Then for every positive choice of $\epsilon$, no matter how small, the domain of the operator contains a function which vanishes outside of an interval of length $\epsilon$ and which does not vanish identically.

The local operator problem is closely related to a problem of Levinson [16] for the growth of entire functions of minimal exponential type along a line. Let $\left(\lambda_{n}\right)$ be a sequence of real numbers with unit density, so that

$$
\lim \lambda_{n} / n=1
$$

as $n \rightarrow+\infty$ and as $n \rightarrow-\infty, n$ integral. Does there exist a nonconstant entire function $K(z)$ of minimal exponential type which remains bounded at the points $\left(\lambda_{n}\right)$ ? He shows that no such function exists if

$$
\left|\lambda_{n}-n\right| \leqq \epsilon(n),
$$

where $\epsilon(x)$ satisfies

$$
\int\left(1+t^{2}\right)^{-1} \epsilon(t) \log |t / \epsilon(t)| d t<\infty
$$

and some smoothness conditions. On the other hand he is able to construct such functions under general conditions in which

$$
\int\left(1+t^{2}\right)^{-1} \epsilon(t)=\infty \text {. }
$$


There is a gap between these two conditions due to a logarithmic factor in the integrand.

It is a general principle in the theory of differential equations that a nontrivial local operator is always unbounded. The principle has a precise formulation in Fourier analysis. An entire function of minimal exponential type is a constant if it remains bounded on the support of a measure whose Fourier transform vanishes in an interval. My approach to Levinson's problem is to construct a step function of bounded variation whose jumps occur in the given sequence $\left(\lambda_{n}\right)$ and whose Fourier transform vanishes in an interval.

Let $\phi(x)$ be a uniformly continuous, increasing function of real $x$ such that

$$
\int\left(1+t^{2}\right)^{-1}|\phi(t)-\pi t|^{2} d t<\infty .
$$

Then for any given number $a, 0<a<\pi$, there exists a nonconstant step function $\mu(x)$, whose jumps occur only at points $x$ where $\phi(x)$ $\equiv 0(\operatorname{modulo} \pi)$, such that $\int e^{i x t} d \mu(t)$ vanishes in $[-a, a]$. The theorem is given in my paper [14] on applications of spaces of entire functions. I have since been able to weaken the hypothesis to

$$
\int\left(1+t^{2}\right)^{-1}|\phi(t)-\pi t| d t<\infty .
$$

If $\lambda_{n}$ is defined so that $\phi\left(\lambda_{n}\right)=n \pi$, then the jumps of $\mu(x)$ occur in the set of points $\left(\lambda_{n}\right)$. If I define

$$
\epsilon(x)=|x-\phi(x) / \pi|,
$$

then

$$
\left|\lambda_{n}-n\right|=\epsilon(n) \text {. }
$$

In this notation my principal hypothesis reads

$$
\int\left(1+t^{2}\right)^{-1} \epsilon(t) d t<\infty .
$$

The result closes a gap in Levinson's book.

Levinson's construction of entire functions of minimal exponential type which are bounded on a given real sequence is difficult and requires more than thirty pages. It is therefore of some interest to note that an existence theorem can be obtained from the above stated results on nonvanishing Fourier transforms. If $K(x)$ is a function which does not satisfy (1), if $|K(x)| \geqq 1$, and if $\log |K(x)|$ is uni- 
formly continuous, then there exists a nonconstant entire function $F(z)$ of minimal exponential type such that $|F(x)| \leqq|K(x)|$ for all real $x$. It follows that if $\left(a_{n}, b_{n}\right)$ are disjoint intervals to the right of $x=1$, if their lengths are bounded away from zero, and if (2) holds, then there exists a nonconstant entire function of minimal exponential type which remains bounded on the real complement of $U\left(a_{n}, b_{n}\right)$.

\section{REFERENCES}

1. A. Beurling and P. Malliavin, On Fourier transforms of measures with compact support, Acta Math. 107 (1962), 291-309.

2. R. P. Boas, Jr., Entire functions, Academic Press, New York, 1954.

3. - Growth of analytic functions along a line, J. Anal. Math. 4 (1954), 1-28.

4. L. de Branges, Local operators on Fourier transforms, Duke Math. J. 25 (1958), $143-154$. 824.

5. - The a-local operator problem, Canad. J. Math. 11 (1959), 583-592.

6. —_ The Stone-Weierstrass theorem, Proc. Amer. Math. Soc. 10 (1959), 822-

7. - The Bernstein problem, Proc. Amer. Math. Soc. 10 (1959), 825-832.

8. - Some mean squares of entire functions, Proc. Amer. Math. Soc. 10 (1959), 833-839.

9. - Some Hilbert spaces of entire functions, Proc. Amer. Math. Soc. 10 (1959), 840-846.

10. - Some Hilbert spaces of entire functions, Trans. Amer. Math. Soc. 96 (1960), 259-295.

11. - Some Hilbert spaces of entire functions. II, Trans. Amer. Math. Soc. 99 (1961), 118-152.

12. - Some Hilbert spaces of entire functions. III, Trans. Amer. Math. Soc. 100 (1961), 73-115.

13. - Some Hilbert spaces of entire functions. IV, Trans. Amer. Math. Soc. 105 (1962), 43-83.

14. - Some applications of spaces of entire functions, Canad. J. Math. 15 (1963), 563-583.

15. M. Kreln and D. Milman, On extreme points of regular convex sets, Studia Math. 19 (1940), 133-138.

16. N. Levinson, Gap and density theorems, Amer. Math. Soc. Colloq. Publ. Vol. 26, Amer. Math. Soc., Providence, R. I., 1940.

17. P. Malliavin, Approximation polynomiale pondéré et produits canoniques, C. R. Acad. Sci. Paris 247 (1958), 2090-2092.

18. R. E. A. C. Paley and N. Wiener, Fourier transforms in the complex domain, Amer. Math. Soc. Colloq. Publ. Vol. 19, Amer. Math. Soc., Providence, R. I., 1934.

19. H. Pollard, The Bernstein approximation problem, Proc. Amer. Math. Soc. 6 (1955), 402-411.

20. M. H. Stone, Linear transformations in Hilbert space and their applications to analysis, Amer. Math. Soc. Colloq. Publ. Vol. 15, Amer. Math. Soc., Providence, R. I., 1932.

21. E. C. Titchmarsh, Eigenfunction expansions associated with second-order differential equations, Clarendon, Oxford, 1946.

Purdue University 